\title{
Rembrandts späte Zeichnungen mit der Rohrfeder - ein für die Veranschaulichung ungeeignetes Mittel?
}

\author{
Werner Busch
}

Die Rembrandt-Caravaggio-Ausstellung 2006 in Amsterdam war in gewisser Hinsicht eine Offenbarung und das, obwohl das Konzept mehr als fragwürdig erschien. ${ }^{1}$ Die Ausstellung nahm ihren Ausgang von dem Faktum, dass der frühe Rembrandt sich intensiv an den Utrechter Caravaggisten orientiert hat. Insofern konnte eine Gegenüberstellung von Frühwerken Rembrandts mit Werken Caravaggios auf einer sehr direkten Ebene Sinn machen und klassische kunsttheoretische Überzeugungen bestätigen: bei beiden Künstlern ein ungeschönter, vermeintlich unmittelbarer Naturzugriff, der auch vor Hässlichkeit, vor allem der Darstellung von Grausamkeit nicht zurückschreckt, scharfe Ausleuchtung mit starken HellDunkel-Kontrasten, große Betrachternähe des Gezeigten mit gelegentlichem Durchstoßen der ästhetischen Grenze, extremer Gefühlsausdruck, bei beiden Künstlern eine relativ glatte und detaillierte Malweise.

So weit, so gut. Bei Rembrandts „Blendung Samsons“ von 1635 und Caravaggios ,Judith und Holofernes“ von 1599-1600 wird gleichermaßen brutal verfahren, das Blut spritzt. ${ }^{2}$ Und die „Opferung Isaaks" haben beide gemalt und auch hier wird an drastischem Zugriff nicht gespart. ${ }^{3}$ Milder geht es verständlicherweise bei den respektiven $\mathrm{Hl}$. Familien $\mathrm{zu}$, doch schon hier gibt es entschieden weniger zu vergleichen. ${ }^{4}$ Ein sich her$a b$ beugender halb verschatteter Joseph findet sich dutzendfach zuvor in der Bildtradition. Seine Zurücknahme ist seiner bloßen Nährvaterfunktion verdankt. ${ }^{5}$ Schon hier wird der Grundfehler der Ausstellung deutlich. Man hat jeweils nach thematisch Verwandtem gesucht. Wenn es eine Verleugnung Petri von Caravaggio gegeben hätte, so hätte man sie neben Rembrandts entsprechendes Bild von etwa 1660 gestellt, so begnügt man

\footnotetext{
1 Kat.Ausst. Rembrandt-Caravaggio (2006).

2 Ebenda Kat. Nr. 9 und 10.

3 Ebenda Kat. Nr. 21 und 22.

4 Ebenda Kat. Nr. 11 und 12.

5 Heublein (1998).
} 
sich mit Caravaggios „Gefangennahme Christi“ wegen der vergleichbaren Halb- bzw. Dreiviertelfiguren, der künstlerischen nächtlichen Beleuchtungssituation und der querformatigen Anlage der vielfigurigen Komposition. ${ }^{6}$ Doch wirklich miteinander zu tun haben die Bilder nichts, selbst wenn „Die Verleugnung Petri“ noch die caravaggeskeste Bildprägung des späten Rembrandt ist, denn einmal noch greift er das Motiv der verdeckten Lichtquelle auf. Die übrigen Vergleiche mit Rembrandts Spätwerk waren schlicht abwegig und erschöpften sich in gänzlichen Äußerlichkeiten, was Rembrandts differenter Malweise verdankt ist, wie der Katalogessay zu Licht und Farbe bei Caravaggio und Rembrandt auch völlig überzeugend konstatiert. ${ }^{7}$ Doch auch vom Aufbau her haben Rembrandts ,Judenbraut“ und Caravaggios „Bekehrung der Maria Magdalena“ keinerlei Berührungspunkte.

Was hat die Ausstellung dennoch so augenöffnend gemacht? Es waren Einzelbeobachtungen, die Rückschlüsse auf die Weise der Bild- und Sinngenerierung zulassen, auf das jeweilige Verständnis von Malerei. Zwar ist der Vergleich von Caravaggios „Büßender Magdalena“ (Abb. 1) von 1598 mit Rembrandts „Flora“ von 1634 schlicht ohne Sinn, ${ }^{8}$ denn man wird nicht behaupten können, dass die Feststellung der Tatsache, dass es sich jeweils um Einzelfiguren handelt und die Gewandmuster detailliert wiedergegeben sind, besonders erkenntnisfördernd ist. Doch schaut man Caravaggios „Büßende Magdalena“ nur lange genug an, so entdeckt man nicht nur die Träne der Büßerin, die sich vom Auge gelöst hat, ja, das durchstochene Ohrläppchen, das noch vor kurzem den am Boden abgelegten Ohrring getragen hat, sondern man realisiert mit einem gewissen Schrecken, dass die dahinschmelzende Verzweiflung besonders dadurch ihren Ausdruck gefunden hat, dass die Dame, um es salopp zu sagen, quasi ohne Unterleib ist. Auch die dicken Gewandbäusche können die unteren Extremitäten nicht sinnvoll verbergen, es ist für sie einfach kein Platz da. Ware ihre anatomisch sinnvolle Erstreckung dargestellt worden, hätte das Bild entschieden an Ausdrucksmacht verloren, vom Zusammengesunkenen wäre durch die Ausfaltung der Beine, aufgrund des Sitzmotives auf dem extrem niedrigen Stuhl, geradezu unschicklich abgelenkt worden. Das Körper- und Sitzmotiv stimmen mitnichten und werden doch für den, der sich der Ausdrucksdimension überlässt, nicht nur nicht moniert,

6 Kat.Ausst. Rembrandt-Caravaggio (2006) Kat. Nr. 16 und 17.

7 van Eikema Hommes, van de Wetering (2006) 164-79, bes. $178 \mathrm{f}$.

8 Ebenda Kat. Nr. 13 und 14. Zu Caravaggios „Magdalena“ s. Krüger (1999) 33-49. 
sondern gar nicht wahrgenommen. Die Ausdruckserfahrung übertönt den Wunsch nach Rationalisierung.

Und dann realisiert man, dass es eben dieses Motiv in gänzlich verwandter Funktion auch bei Rembrandt gibt, und zwar gerade im Spätwerk. Insofern wäre es gut gewesen, die „Magdalena“ mit dem Kassler „Jakobssegen“" von 1656 (Abb. 2) zu vergleichen. ${ }^{9}$ Auch hier verschleiert der große, schwere rote Bettüberwurf ebenfalls die nicht vorhandene Andeutung von Jakobs Unterleib. Keinesfalls kann er so im Bett sitzen, wie Rembrandt ihn uns zeigt und doch ist er gänzlich überzeugend, nichts lenkt vom zentralen Brüderpaar Ephraim und Manasse in seiner Schoßbeuge, vom Vorgang des überraschend an den Jüngeren erteilten Segen ab. Joseph, der vorsichtig versucht, die Hand Jakobs zu steuern, kann das Wirksamwerden von Gottes Ratschluss nicht verhindern. Und selbst wenn auch die Bettperspektive nicht ,stimmt“, Manasse hinter Ephraim eigentlich gar keinen Platz hat, so ist das Ganze in seiner Wirkung doch gänzlich überzeugend. Wie kann es zu einer derartigen parallelen Bilderfindung bei Rembrandt und Caravaggio kommen? Wie kann es sein, dass sie Bildwirksamkeit gegen Bildrichtigkeit ausspielen (können)? Was lässt sie derartige Verfahren der Ausdruckssteuerung - und es gibt ihrer mehrere überhaupt entdecken?

Man kann es mit einem Wort sagen: der Werkprozess, oder besser, der Bildschöpfungsprozess, ihre Produktionsweise. Denn in einem stimmen Caravaggio und Rembrandt überein: sie erfinden unmittelbar auf der Leinwand, verfolgen keinen klassischen Werkprozess und das heißt vor allem, es gibt bei ihnen keinen zeichnerischen Entwurf, in verschiedenen Stufen ausgereift nach dem akademischen Verfahren: erste Ideenskizze, folgender Kompositionsentwurf, Studien zu Einzelfiguren am Modell, endgültiger zur Übertragung auf die Leinwand bestimmter, womöglich quadrierter Entwurf oder Karton, der die Bilderfindung voll- und endgültig in sich fasst, so dass die Ausführung im Gemälde primär eine farbige Materialisierung der vorentworfenen Idee darstellt. Dagegen bei Rembrandt und Caravaggio mit dem Pinsel unmittelbar auf der Grundierung und zumeist monochromen Imprimitur mehr oder weniger flüchtig festgehaltener Entwurf als gewisse Anleitung für die sogleich darüber angelegte farbige Malerei. Mehr als eine Anleitung zur Grobverteilung kann der Entwurf nicht sein, denn er wird ja im Ausführungsprozess sofort überdeckt. Caravaggio dürfte gelegentlich auch ganz auf sog. „abbozzi“

9 Kat.Ausst. Rembrandt Rembrandt (2003) Kat. Nr. 32. 
verzichtet haben. Dieses Verfahren erklärt die zahlreichen Pentimenti bei Caravaggio wie Rembrandt. Anders ausgedrückt: die Vorstellung davon wie das Bild werden soll, bildet sich erst schrittweise im Prozess der malerischen Anlage, praktische Entscheidungen führen zu Folgeüberlegungen, Folgeüberlegungen können zuvor Angelegtes wieder aufheben, Korrekturen notwendig werden lassen, der Prozess kann stocken, der Maler kann sich verrannt haben, findet bessere Lösungen etc.

Nun hat die Forschung ein solches Procedere nicht leicht akzeptieren können, auch im Falle von Caravaggio und Rembrandt nicht, wobei deren Ausgangslage in einem Punkt radikal verschieden ist: von Caravaggio haben wir keine einzige Zeichnung, von Rembrandt Hunderte. Das hat die neuere Caravaggio-Forschung nicht ruhen lassen. In einer Arbeit von 2002 wird mit allem Nachdruck ein verloren gegangenes zeichnerisches Oeuvre von Caravaggio vorausgesetzt, mit einer vermeintlich zwingenden Begründung: zahlreiche Bilder Caravaggios folgten einer klaren geometrischen, auf bestimmten Verhältnissen gegründeten Ordnung, sie schließe ein „Probieren“ auf der Leinwand aus. ${ }^{10}$ Und die auf vielen Gemälden unmittelbar - besonders bei Streiflicht - sichtbaren ,incisioni“, die Ritzungen in die noch feuchte Grundierung, werden nicht als eine Formfixierung allein auf der Leinwand angesehen, die die Grisailleanlage, den Chiaroscuro-abbozzo überflüssig macht, sondern als genaue Übertragungen seiner Vorzeichnungen, zu sicher und weitgehend seien die Formvorgaben. Wie hätte, so wird argumentiert, der Künstler den Ort abstrakter geometrischer Figuren so präzise finden können? Die Ritzungen als Ergebnis von Übertragungen hätten zudem den Vorteil, dass sie auch bei darüber gelegter farbiger Fassung noch durchscheinen, und die Vorstellung der Gesamtfiguration auf diese Weise nicht verloren ginge. ${ }^{11}$ So ernst man derartige Überlegungen nehmen muss, denn die ,incisioni“" scheinen ausgesprochen sicher angelegt, so geht doch kein Weg daran vorbei, dass jegliche Zeichnungen fehlen. Dies Caravaggios unstetem Lebenswandel zuschreiben zu wollen, der von Flucht und Verfolgung geprägt gewesen sei, erscheint doch als zu kurzschlüssig. ${ }^{12}$ Caravaggios einflussreiche Gönner sollten nicht eine Zeichnung verehrt bekommen haben, wenn sie denn existiert hätten? Und zudem: eine geometrische Ordnung ist eine Flächenordnung, von der genauen Erstreckung des Bildkörpers bedingt

10 Kroschewski (2002) bes. Kap. II; VII; VIII.2; XII, mit Referat der gesamten Forschung.

11 Ebenda Kap. VIII.2; X.

12 Ebenda $70 \mathrm{f}$. 
und auf diesem mit wenigen Angaben zu markieren, so dass nichts dagegen spricht, dass die Formfindung auf der Leinwand selbst auf der Basis abstrakter Vorgaben stattgefunden hat. ${ }^{13}$

Wie aber nun verhält es sich mit den vielen hundert Zeichnungen von Rembrandt? Es hat die Forschung immer verwundert, dass man nur gut zwanzig Zeichnungen davon ganz direkt mit bestimmten Werken in Verbindung bringen kann. Lange wurden wenigstens diese als direkte Vorzeichnungen angesehen. Die neuere Zeichnungsforschung zu Rembrandt, die inzwischen zu so gut wie allen bedeutenden Sammlungen mit Rembrandt-Zeichnungen Bestandskataloge hervorgebracht hat, lässt davon zur eigenen Verwunderung in der Funktion als Vorzeichnung so gut wie keine einzige Zeichnung übrig. Die allermeisten sind während des Malprozesses entstanden, dann, wenn Rembrandt in seinem Bildfindungsprozess stecken blieb, ihm grundsätzliche Änderungen als unausweichlich erschienen und er sich zeichnerische Gedanken über das weitere Vorgehen machen wollte. Deswegen geben diese Zeichnungen zumeist in ausgesprochen flüchtiger Form den Stand der Bildprägung, an dem Punkt, als die Arbeit ins Stocken geriet, wieder, um dann mit wesentlich energischeren Strichen in Hinblick auf eine grundsätzliche Veränderung überzeichnet zu werden. Röntgenfotos bzw. Infrarotreflektographien der Gemälde, die frühere Zustände dokumentieren können, machen diese Einsicht unhintergehbar.

Unmittelbar nachvollziehbar, weil in zwei Bilder zerlegt, ist der Veränderungsprozess im Falle des „Abrahamsopfer“ (Abb. 3 und 4). ${ }^{14}$ Die ursprüngliche, gänzlich von Rembrandts Hand stammende Fassung in St. Petersburg von 1635 wurde in der Münchner, mit Hilfe von Rembrandts Werkstatt erstellten Fassung grundsätzlich revidiert, vor allem, was den eingreifenden Engel angeht. Rembrandt hat es am unteren Rand dieses Bildes vermerkt: „,Rembrand. verandert. En overgeschildert 1636“, „Von Rembrandt geändert und übermalt, 1636“. ${ }^{15}$ Die Infrarotreflektographie des Münchner Bildes macht deutlich, dass auf der Leinwand sich ursprünglich eine vollständige Kopie der St. Petersburger Fassung befunden hat. ${ }^{16}$ Sie hat Rembrandt als Ausgangspunkt genommen - ein Verfahren im Üb-

13 Zur geometrischen Bildordnung: ebenda Kap. X und XI.

14 Kat.Ausst. Rembrandt. Die Opferung Isaaks (2004).

15 Ebenda 48-54 und 57-59.

16 Ebenda 54 und Abb. 24. 
rigen, das für mehrere Bilder Tizians nahezu identisch überliefert ist. ${ }^{17}$ Die Kopie des St. Petersburger Bildes dürfte in der Anlage Werkstattarbeit gewesen sein. Dann hat Rembrandt sich Gedanken gemacht, wie er das Eingreifen des Engels noch überzeugender machen könnte. Wenn er in der ersten Fassung von der Seite kam, und trotz Wolkenverhüllung wie unter dem Oberkörper abgeschnitten erschien, so taucht dieses Problem, wenn er quasi von hinten in extremer Verkürzung sich nähert, nicht auf. Dies entwarf Rembrandt in einer Zeichnung in roter und schwarzer Kreide mit grauer Lavierung (Abb. 5). Sie ist passagenweise so unartikuliert, dass man sie Rembrandt meinte absprechen zu sollen. ${ }^{18}$ Doch unartikuliert sind diejenigen Partien, die nicht weiter verändert werden sollten, sie brauchten keine Präzisierung, dagegen ist das Engelsmotiv spezifischer und auch das letztlich dann wieder verworfene Motiv mit dem entfallenen Dolch - er verliert besonders in der präzisierten Fassung als mit umgekehrter Schneide zur Erde fallend seine Bedrohlichkeit - ist gut nachvollziehbar. Das Opferfeuergefäß der ersten Fassung, das ursprünglich vom thematischen Vorbild Lastman stammt, ${ }^{19}$ hat auch noch in der Zeichnung am rechten Rand sein Vorkommen, in der endgültigen zweiten Fassung ist es verschwunden, dafür ist das Ersatzopfer, der Widder, deutlich hervorgehoben. Das macht Sinn, denn das Feuer kommt ja, durch das Eingreifen des Engels, nicht zum Einsatz.

Diesen Typus der intermediären Korrekturzeichnung gibt es bei Rembrandt von allem Anfang an bis in die späteste Zeit, vom ,Judas“ aus dem Jahr 1629 bis zu den „Staalmeesters“, die 1662 datiert sind. ${ }^{20}$ Es gibt ihn auch, wenn auch seltener, im Zusammenhang mit der Radierung, besonders nachdrücklich bei der "Grossen Judenbraut" von 1635. Nach den ersten beiden Zuständen (Abb. 6), die nur Kopf, Schultern, die gewaltige Haarpracht und den Hintergrund zeigten, noch nichts von Armen, Händen, Gewand und unter den schweren Falten verborgenen Beinen, legte

17 Der Kat.Ausst. Der späte Tizian und die Sinnlichkeit der Malerei (2007) ist weitgehend diesem Verfahren gewidmet.

18 Kat.Ausst. Rembrandt. Die Opferung Isaaks (2004) 55-57 und farbige Abb. 28 und Kat. Drawings by Rembrandt and his Circle (1992) Kat. Nr. 13, 56-58.

19 Waiboer (2006) 45 und 42, Abb. 3; Tümpel (1991) 65-67.

20 Kat. Tekeningen van Rembrandt (1985) Kat. Nr. 5, 12-15; van de Wetering (2000) 75-81, Abb. fig. 103-106 (,Judas“); Kat. Tekeningen van Rembrandt (1985) Kat. Nr. 56, 123-125 (,,Staalmeester““); Royalton-Kisch (1989) 128-145, 130-133 (,Judas“), 142-144 (,Staalmeesters“). 
Rembrandt eine Zeichnung von der Größe der Radierung an (Abb. 7). ${ }^{21}$ Den Bestand des Zustandes der Radierung hielt er mit ausgesprochen dünnen, hellen Federstrichen fest, um in einem deutlich dunkleren Ton und mit sehr viel breiteren und kühneren Federstrichen, die kaum eine präzise Formvorstellung zulassen, die denkbare Weiterentwicklung der Radierung zu entwerfen: die Hand mit der Schriftrolle, die andere, die sich auf die Lehne des Stuhles stützt und - erstaunlich hellsichtig - die beiden tiefen Schüsselfalten, die den jeweils auf den Knien auflastenden schweren Gewandbausch markieren. Gerade dies letztere, das als Idee in die Radierung übertragen wird, folgt nicht etwa klaren anatomischen Vorstellungen, vielmehr wird durch die starken Licht-Schatten-Kontraste eine gewisse Vorstellung von Körperlichkeit evoziert, ohne wirklich gezeigt zu sein. Rembrandt genügen die wirren Strichbündel als Anregung zur Umsetzung in die Radierung.

Der Zusammenhang von Frühwerk und Spätwerk ist auch darin zu sehen, dass Rembrandt nicht selten Themen des Frühwerkes aufgreift, von der damaligen Bildfindung ausgeht, sie in Grenzen aufbewahrt, man könnte sagen, dialektisch aufhebt, den Ausgangspunkt also braucht, um dennoch etwas Neues mit gänzlich gewandelter Ausdrucksdimension daraus zu machen. Diese neue Ausdrucksdimension ist jeweils medienbedingt, oder anders ausgedrückt, Rembrandt wählt Medienmittel in Zeichnung, Druckgraphik und Malerei, die aus ihrer medialen Bedingtheit heraus bestimmten Ausdrucksbereichen gegenüber offen, anderen gegenüber verschlossen sind. Aber es sind auch die gewählten Medien selbst, die Ausdruckstendenzen hervortreiben.

Bevor dies exemplarisch an den späten Rohrfederzeichnungen verfolgt werden soll, einige hinführende Bemerkungen. Das Thema des „Abrahamsopfers" greift Rembrandt in den fünfziger Jahren wieder auf. Zuerst in einer Zeichnung von Anfang der fünfziger Jahre (Abb. 8). ${ }^{22}$ Und so sehr hier die gänzliche Motivverwandlung auffällt, es geht immer noch darum, eine überzeugende Form für das Eingreifen des Engels zu finden. Nicht mehr das plötzliche Hereinbrechen des Engels ist Thema, er fällt Abraham nicht mehr in den Arm, vielmehr erscheint er in einem breiten Lichtband, neigt sich verständnisvoll zu Abraham herab, der auf zittrigen Beinen nur mit Mühe in der Lage scheint, Gottes Gebot zu folgen. Isaak liegt gefesselt

21 Kat.Ausst. Rembrandt the Printmaker (2000) Kat. Nr. 25 zum 2. und 4. Zustand und zum Status der Zeichnung (fig. a), 140-144.

22 Kat.Ausst. Rembrandt. Die Dresdener Zeichnungen (2004) Kat. Nr.112, 197-199. 
rücklings auf dem Opferstein. Abraham hat mit verkrampfter Hand seinen Kopf zurückgebogen, um den Hals freizulegen und zum Opfer schreiten zu können. Der Engel erlöst ihn hiervon, indem er ihm einfach die Hand auf das Greisenhaupt legt. Nichts ist mehr wirklich durchgebildet, die Dinge sind nur mit wenigen Strichen angedeutet, die selten eine geschlossene Form bilden. An Abrahams labilem Standmotiv sind Korrekturen vorgenommen worden. Wie oft bei Rembrandt bleiben sie nebeneinander stehen, es geht nicht um die Fixierung, sondern um die Evokation von Form.

Diese Zeichnung geht Rembrandts letzter Fassung des Themas voran, der Radierung von 1655 (Abb. 9). ${ }^{23}$ Sie ist von einer Klassizität, wie sie sich Mitte der fünfziger Jahre vielfach bei Rembrandt findet, das Phänomen wird bei der Behandlung der Rohrfederzeichnungen zu charakterisieren sein. Engel, Abraham und Isaak sind nun zu einer blockhaften Gruppe zusammengefasst. Isaak kniet geradezu demütig, nicht mehr verkrampft gefesselt. Abraham hat mit breiter Hand sein Gesicht bedeckt, das Messer in der anderen Hand weit von sich gestreckt. Der Engel greift ganz körperlich ein, aber nicht plötzlich von oben. Er hat Abraham gänzlich umfangen. Seine eine Hand umfasst Abrahams Gelenk, in gewisser Hinsicht immer noch das alte Motiv, das es nicht nur in Rembrandts Frühwerk gibt, sondern in der Bildtradition schon lange zuvor. Aber es ist weniger ein rasches Eingreifen als eine mit dem ganzen Körper vorgenommene, Abraham aus seinem Schicksal nehmende beruhigende Kraftübertragung. Die andere Hand drückt, nicht gewaltsam, Abrahams Arm mit dem Dolch an den Körper. Abraham wendet den Kopf zum nahen Kopf des Engels, offenbar erkennt er Gottes neuen Beschluss seinem treuen Knecht gegenüber.

Dieser Moment der Kraftübertragung, die den Erkenntnisakt auslöst, ist von Rembrandt später bei seinem ,Jakob ringt mit dem Engel“" von 1659 noch einmal zum Thema gemacht worden, wiederum bei einem Thema, das eigentlich einen dramatischen Moment fordert. Denn Jakob, der den Engel im Kampf nicht lassen will, so dass dieser ihm schließlich die Hüfte zerbricht, das Beinmotiv des Engels deutet es an, Jakob ist eher in einem somnambulen Zustand und der Engel schaut ihn sinnend an. Wieder ist der dramatische Akt im Erkenntnismoment aufgehoben, der Gruppe eine statuarische Monumentalität beigesellt worden. Nicht anders beim gleichzeitigen „Moses“ (Abb. 10), ebenfalls in Berlin, bei dem offen

23 Kat.Ausst. Rembrandt the Printmaker (2000) Kat. Nr. 77. 
bleibt, ob er die Gesetzestafeln zerschmettert oder als alles änderndes Gotteszeichen in die Höhe hält. Nicht Handlungsvollzug ist gezeigt, sondern ein Moment stillgesteller Handlung, die es auch dem Betrachter ermöglicht, den tieferen Sinn des Vorganges zu reflektieren. In beiden Fällen, in extremer Weise beim „Moses“, ist die Farbmaterie in großen Partien kaum gestaltet, in der Untermalung stehengeblieben. Verdichtet ist sie nur in den Lichtpartien, beim „Moses“ auf den Armen und vor allem auf der von Gott erleuchteten Stirn. Hier ist das reliefbildende Impasto eindeutig am dicksten und damit ganz wörtlich genommen, aber eben auch im übertragenen Sinn gemeint, am hervortretendsten. ${ }^{24}$ Dieses Nebeneinander von Gestaltetem und Ungestaltetem hat durchaus auch sein Äquivalent in der Zeichnung, aber auch in der Druckgraphik. Wenn Rembrandt in der Zeichnung der späten vierziger Jahre schrittweise vom Gänsekiel, der den freien flüssigen Verlauf der Linie zulässt, jeder Wendung problemlos folgt und den Künstler vor allem in den dreißiger Jahren zu überschüssigen Äußerungen in Form ornamentaler Schnörkel verlockt, zur Rohrfeder überwechselt, die alles andere als problemlos über das Papier läuft, vielmehr kratzt und kleckert, jeden schönheitlichen Linienfluss unterbindet, mal mehr oder weniger Farbe abgibt, so dass die Linie jeder Gleichmäßigkeit entbehrt, die den Künstler veranlasst, eher eckige kubische Kompartimente zu kombinieren, grobe, kaum einem Formvorbild folgende, nicht selten geradezu hässliche, verzerrende, reduzierte, nur entfernt Ähnlichkeit evozierende Gebilde hervorbringen, ${ }^{25}$ dann ist das Äquivalent in der Druckgraphik die Wahl der Kaltnadel. ${ }^{26}$ Diente sie zuvor bei Rembrandt dazu, nach ersten Probeabzügen der Radierungen den Vordergrund zu stärken, ein Repoussoir zu markieren und damit Raum zu schaffen, da ihre groben, ins Metall gerissenen und so zu den Rändern hin ausfran-

24 Zu den Berliner Bildern: van de Wetering (2000) 207-210 (,Moses“); Kat.Ausst. Rembrandt. Genie auf der Suche (2006) Kat. Nr. 70 („Moses“), 71 (,Jakob“).

25 Allein zwei Arbeiten scheinen mir dem Phänomen der Rohrfeder wirklich näher zu kommen: Frey (1959) 91-116 (generell zum Fragmentarischen im Spätwerk); Courtright (1996) 485-510 (sie reflektiert intensiv über die Frage der Angemessenheit der Kategorien „vollendet“ - „unvollendet“, die antike Rechtfertigung des „Unvollendeten“, seine rhetorische Dimension, vor allem aber die anti-klassische Dimension und rechtfertigt den ungeschmückten Stil in christlichaugustinischer und protestantischer Tradition. Die „raue Manier" ist, wie auch in diesem Versuch, als Form der „natura naturans“ der entstehenden Formgebung, nicht der vollendeten Form, „natura naturata“, zu verstehen).

26 Der Zusammenhang zwischen Rohrfederbenutzung und Kaltnadelverwendung gesehen bei: Rosand (2002) vor allem 243-245. 
senden und Grate aufwerfenden Linien mehr Farbe aufnehmen, als die bloßen in den Ätzgrund gezeichneten Radierlinien, so emanzipiert sich die Kaltnadelradierung in den fünfziger Jahren und kann bei den „Drei Kreuzen“ von 1653 und dem „Ecce Homo“ von 1655 zum alleinigen Medium werden, das Rembrandt zu geradezu wilden, schier die Platte zerstörenden Zustandsveränderungen nutzt. Die „Drei Kreuze“ ertrinken im Dunkel (Abb. 11), das „Ecce Homo-Blatt“ eröffnet einen Abgrund, vor dem der Betrachter keinen sicheren Ort gewinnen kann. ${ }^{27}$ Zudem gibt es, besonders beim „Ecce Homo“, Partien, deren bloßer Entwurfscharakter offensichtlich ist, den Rembrandt jedoch, trotz der vielen Überarbeitungen, nicht weitergeführt hat. Sollte es sich nicht beim „Moses“, den auch die gesamte neuere Forschung für , unvollendet“ erklärt, um ein ähnliches Phänomen handeln, bei dem die Kategorien „,vollendet“ und ,unvollendet" schlicht falsch gewählt sind? ${ }^{28}$

Auch hier fragt sich, warum Rembrandt ein Medium wählt, das der Strichführung Widerstände entgegen setzt, das kratzt, das Metall eher verletzt als verziert und warum er in beiden Fällen, um es so zu sagen, nicht aufhören kann, die Platte zu verändern, ferner, warum er die Spuren vorheriger Zustände nicht vollständig abschleift, letztlich damit das Werk als unabgeschlossen deklariert. In früheren Zeichnungen kombiniert Rembrandt Federzeichnung in feineren Gänsekiellinien und Pinsellavierung, wobei die Pinsellavierung mitnichten bloßen Flächen- bzw. Schattenton anzugeben hat, ja, es ist geradezu ein Zeichen für nicht vorhandene Eigenhändigkeit, wenn die Lavierung der bloßen Abtönung dient, wie in der Bildtradition üblich. ${ }^{29}$ Vielmehr hat die Pinselzeichnung in mehr oder weniger ausgeprägtem Maße die Funktion, gegenständlich zu bezeichnen, selbständig Form zu bilden.

Ein einschlägiges Beispiel dafür, das gut auch den Entwurfsprozess verdeutlichen kann, ist die Münchner Zeichnung, die zumeist den, im übrigen nicht gänzlich zwingenden Titel trägt „Saskia im Bett mit einer Amme " (Abb. 12) und in die späten dreißiger Jahre zu datieren ist. ${ }^{30}$ Zuerst hat Rembrandt mit der feinen Feder die melancholische, offenbar kranke Frau im Bett mit Kissen und Laken gezeichnet. Schatten, die durch das

27 Kat.Ausst. Rembrandt the Printmaker (2000), Kat. Nr. 73 („Drei Kreuze“), 197-204; Kat. Nr. 78 („Ecce Homo“), 316-322.

28 Zum „Unvollendeten“ des „Moses“: van de Wetering (2000) 207; Kat.Ausst. Rembrandt. Genie auf der Suche (2006) 384.

29 Kat. Drawings by Rembrandt and his Circle (1992) 15 (Royalton-Kisch).

30 Kat. Rembrandt auf Papier (2001) Kat. Nr. 17. 
relativ starke Licht von links, wo wir uns das Fenster zu denken haben, geworfen werden, sind mit Schraffuren angegeben. Dann hat Rembrandt mit stark verdünntem Bister mehr oder weniger die gesamte Fläche des Blattes leicht abgetönt. Ausgeschlossen davon hat er Haube und Arm der Frau, den rechten Teil des Kissens und die obere Partie des Betttuches. Sie erscheinen weiß, konzentrieren unseren Blick geradezu unmerklich auf diesen Bereich, was dem direkten Blick der müden Frau auf uns korrespondiert. Dann hat Rembrandt mit dem Pinsel in leicht dunklerem Ton erst die Amme oder Warterin vor dem Bett auf erstaunlich rasante Weise grob markiert, nichts ist wirklich im Detail ausgeführt, vieles haben wir zu projizieren. Der Schwung, der in der Breite ständig variierenden Pinsellinien, die gelegentlich von der Linie zur lavierenden Fläche werden, dient offenbar dem Künstler im Prozess der Ausführung dazu, nur halb bewusste Entscheidungen zu treffen, eine Linie treibt die nächste hervor, um eine Gestaltandeutung zu ermöglichen. Da die Gestaltandeutung sich nicht zur definitiven Form verfestigt, bleibt das Prozessuale anschaulich. Abschließend deutet Rembrandt mit ein paar unartikulierten Parallelstrichen den Bettbaldachin an und setzt den mit starker oder leicht verdünnter Farbe erfüllten Pinsel mit seinem ganzen feinen Borstenkörper auf die Fläche, um größere Schattenpartien zu markieren. Dadurch ist die im Bett Sitzende von allen vier Seiten gerahmt, was den Fokus auf sie noch einmal verstärkt. D.h.: obwohl die Amme durch die dunklen breiten Linien betont ist und vorne erscheint, ist sie dennoch bloßes Repoussoir. Dass ihr Binnenzeichnung und Detail fehlen, verstärkt für unsere Wahrnehmung, die so nicht längerfristig gefesselt wird, diese Funktion.

Der Entstehungsprozess einer derartigen Zeichnung mitsamt seiner Bedeutung generierenden Funktion wurde deswegen in einiger Ausführlichkeit beschrieben, weil er, trotz seiner teilweise flüchtigen, teils rasanten, teils bloß andeutenden Markierungsweise im Endeffekt Bildmäßigkeit erreicht und zwar, so sonderbar das klingen mag, in erstaunlicher Parallele zum Ölbildprozess. Das, was sie verbindet, ist das Prinzip des "houding". ${ }^{31}$ Folgt man den kunsttheoretischen Quellen des holländischen späteren 17. und früheren 18. Jahrhunderts, dann meint "houding", das wörtlich mit „Haltung“ zu übersetzen wäre, den Aufbau eines Bildes mit tonalen und farbigen Mitteln, wobei diese Mittel technisch geschickt verwendet Wahrnehmung steuern können, vor allem aber, zugespitzt ausgedrückt, durch einen Verzicht auf die Verwendung klassischer, gesetzmäßiger Perspekti-

31 Taylor (1992) 210-232. 
ve und Anatomie dennoch Raum- und Körperillusion zu stiften in der Lage sind. Die Dinge erscheinen richtig, da sie mit Hilfe unserer Projektion die Vorstellung von Räumlichkeit und Körperlichkeit evozieren können, sie provozieren Erfahrungen gerade in ihrem bloß andeutenden Charakter. Letztlich - wie weit es theoretisch reflektiert wird, bleibe dahingestellt, Svetlana Alpers Annahme einer weitgehenden Fundierung der holländischen Kunst des 17. Jahrhunderts durch wissenschaftliche Optik ist umstritten $^{32}$ - ist Malpraxis auch immer eine Anwendung von Wahrnehmungserfahrungen und Wahrnehmungsgesetzen. In der Ölmalerei - und auch dies ist Teil des Konzeptes von "houding" - wird Wahrnehmung und Wahrnehmungskonzentration auch durch die Steigerung des Farbreliefs, durch zunehmendes Impasto, von hinten nach vorne bestimmt. Aus dunkler glatter, nicht selten durchscheinender Hintergrundsfolie, die also den Ton der Imprimitur mitwirken lässt, wächst langsam der Farbkörper heraus, der sein höchstes Relief ganz wörtlich genommen, in den hellsten die Aufmerksamkeit anziehenden Partien findet, hier sind mit deckendem Weiß abschließend die Lichter aufgesetzt. In der Zeichnung leistet das nicht getönte Weiß des Papieres diesen Dienst, ferner das sorgfältig bedachte Verhältnis von detaillierten und bloß andeutenden Partien.

Davon nun allerdings unterscheiden sich tendenziell die späteren Zeichnungen in Rohrfedermanier. Es könnte sein, das Rembrandt die Idee, die Rohrfeder als bevorzugtes Zeichenmedium ab den vierziger Jahren zu benutzen, aufgrund einer Nutzungsfunktion eines bestimmten Zeichentypus gekommen ist. Offenbar schien Rembrandt die Rohrfeder geeignet, bei „modelli“ Verwendung zu finden. Jedenfalls wählt Rembrandt die Rohrfeder, als er einen ersten Gesamtentwurf für seine Radierung mit dem Bildnis des Jan Six von 1647 in etwa im Format der späteren Radierung fertigte (Abb. 13). ${ }^{33}$ Ganz offensichtlich hatte Jan Six die Vorlage gewünscht und dann auf einer ganzen Reihe von Änderungen bestanden. Kurz gesagt: der erste Entwurf, der Jan Six in sehr salopper informeller Pose zeigt, bei der der Hund freudig an ihm hochsprang, sollte transferiert werden in das Bildnis eines Gelehrten. Selbst wenn die informelle Fassung mit Hilfe von Baldessare Castigliones „Il Cortegiano“ von 1528, den Six nachweislich kannte und der auch auf sein späteres gemaltes Porträt von Rembrandt aus dem Jahre 1654 abgefärbt hat, zu rechtfertigen war, indem das Informelle als höfisches Verhaltensideal einer eleganten Leichtigkeit, „sprezzatura“, verstanden wurde, so war Six offenbar doch der offizielle

32 Alpers (1983) bes. Kap. 2.

33 Kat.Ausst. Rembrandt. Der Meister und seine Werkstatt (1991) Kat. Nr. 23. 
Charakter der Radierung wichtiger, die sein Selbstverständnis und seinen intellektuellen Anspruch spiegeln sollte (Abb. 14). ${ }^{34}$ Bücher, Manuskripte, Kunst hatten aufzutauchen, und der Dargestellte, zwar immer noch an die Fensterbank gelehnt, erschien bei konzentrierter Lektüre. Eine besondere Rolle spielt in der Radierung die Beleuchtung, offensichtlich zu verstehen als Erleuchtung: der Raum ist weitestgehend in Dunkel getaucht, nur weniges empfängt ein wenig Licht oder leuchtet im Dunkel auf. Doch das offene Fenster liefert strahlende, geradezu absolute Helligkeit, sie spendet dem schräg Stehenden Leselicht. Offenbar wird das Licht der angestrahlten Manuskriptseite im Gesicht von Jan Six reflektiert, lässt Gesicht und Haar im Licht geradezu transparent werden. Rembrandt scheint beständig mit Reflexlicht experimentiert zu haben, an Ärmeln und vor allem den Händen ist es hier besonders gut zu verfolgen.

Davon hat die Zeichnung noch wenig. Lichtführung und Attribute dienen in der Radierung dem Gelehrtenkonzept. Was dann liefert die Zeichnung? Offenbar zweierlei: eine generelle Vorstellung, ohne allzu viel Konkretion, die weitere Sinnzuschreibung zulässt, auch von Seiten des Dargestellten, und eine Vorstellung von der malerischen Fassung des Endproduktes, ohne auch hier Festschreibungen vorzunehmen. Insofern ähnelt dieser zeichnerische „modello“ einem malerischen „,bozzetto“, fungiert dadurch, dass er bereits eine Vorstellung ermöglicht, als Gedankenanregung. Um diese Doppelfunktion zu erfüllen, ist die Rohrfederzeichnung besonders geeignet. Deutet sie doch nur an, ist in ihrer sperrigen Form nur auf dem Weg zur ,eigentlichen“ Form. Die Kratzspuren, die sich auch innerhalb der einzelnen Linie finden, lösen diese tendenziell wieder auf, lassen sie als vorläufig erscheinen. Da kaum eine Linie für sich steht und somit als definitive Bezeichnung zu lesen wäre, sondern mehrere interagieren, korrigierend übereinander gelegt werden, nur selten von Deckweiß ungeschehen gemacht werden - immer dann, wenn sie keine Sinnvorstellung mehr zulassen oder aber aufgrund grundsätzlicher Neuorientierung im Wege stehen ${ }^{35}-$, da das so ist, hat die Zeichnung

$34 \mathrm{Zu}$ Jan Six, seinem Bildnis von 1654, dem Castiglione-Verweis: de Jongh (1985) 67; Smith (1988) 42-43; van de Wetering (2000) 160-162, daran anschließend der Vergleich Rembrandt - Tizian und zu ihrer „pittura di macchia“ und ihren „pentimenti“, 162-169. Zur Radierung: Kat.Ausst. Rembrandt. Der Meister und seine Werkstatt (1991) Kat. Nr. 23; Kat.Ausst. Rembrandt the Printmaker (2000) Kat. Nr. 57, 235-243. Zur Rolle der Zeichnung für die Druckgraphik: RoyaltonKisch (2000) 64-81, zu ,Jan Six“ 67-69.

35 Z. B. Kat. Tekeningen van Rembrandt (1985) Kat. Nr. 46. 
den Status des Vorschlages, mehr noch, sie fordert zur Fortschreibung, Korrektur, Konkretion auf. Anders ausgedrückt: sie ist in der Bosse stehen geblieben, Feinarbeit hat noch nicht stattgefunden. So wie ein Gliedermann, ein Manichino, jede Haltung einnehmen kann, ohne sie anatomisch korrekt vorzustellen, so liefert die Rohrfederzeichnung Anhaltspunkte für eine Vorstellung.

In den fünfziger Jahren, als Rembrandt die Möglichkeit der Rohrfederzeichnung auslotet, verwendet er sie offenbar noch ein paar Mal, um eine erste Idee für ein Porträt zu markieren, die der weiteren Bildfindung Vorschub leistet. Zwei Zeichnungen in Stockholm und eine im British Museum nutzen offenbar Hendrickje Stoffels als Modell für eine derartige Anregung. ${ }^{36}$ Wie sehr Rembrandt über die medialen Bedingungen und Möglichkeiten nachdenkt, das mag auch die Wahl der Papiere für eine ganze Reihe von Rohrfederzeichnungen verdeutlichen. Besonders geeignet schien ihm offenbar ein ausgesprochen grobfaseriges, bräunliches Papier mit körnigen Einsprengseln, das der kratzigen, manchmal, wenn bewusst wenig Farbe aufgenommen wurde, bröckeligen Erscheinung des Rohrfederstrichs korrespondiert. Ein einprägsames Beispiel stellt die Dresdener Zeichnung einer Frau mit pissendem Kind dar (Abb. 15), zu der eine Variante in Amsterdam, offenbar vom selben Papierbogen, existiert. ${ }^{37}$ Hier ist die für die fortgeschrittene Entwicklung der Rohrfederzeichnung typische Unterteilung der Körperformen in kubische Kompartimente bereits vollständig vorhanden, wir hatten gesagt, dass sie der Klassizität zuarbeitet, und sie kommt besonders durch die Frontalanordnung der kleinen Figurengruppe zur Wirkung. So banal das Motiv erscheint, es gewinnt monumentale Züge. Vieles ist kaum noch vermerkt und dennoch für die Formvorstellung anwesend wie die Hände und Füße der Frau, die doch mit ihren Händen das Kind hält und das Hemdchen hochschiebt. Man betrachte nur die linke Schulter der Frau auf der Dresdener Zeichnung und die rechte Schulter der Frau der Variante in Amsterdam. Die Rohrfederlinie folgt nicht etwa dem Verlauf der Schulter, sondern setzt mehrfach an, verläuft unregelmäßig, geradezu staubig, Farbe abgebend in kantigen Bahnen. Erst die Addition mehrerer dieser geraden Linienfragmente er-

36 Kat.Ausst. Rembrandt. Der Meister und seine Werkstatt (1991) Kat. Nr. 35, 35a; Kat. Drawings by Rembrandt and his Circle (1992) Kat. Nr. 59.

37 Kat.Ausst. Rembrandt. Die Dresdener Zeichnungen (2004) Kat. Nr. 115; Kat. Tekeningen van Rembrandt (1985) Kat. Nr. 49. Gelegentlich verwendet Rembrandt dieses „Hafergrützenpapier“ auch für Landschaftszeichnungen, s. ebenda Kat. Nr. 35 (vergl. auch Kat. Nr. 35a und b), 51. 
möglicht eine Formvorstellung. Wuirde man die Schulterpartie im Ausschnitt vergrößern, sie würde in ihrer bezeichnenden Funktion nicht mehr zu erkennen sein. Hier scheint das Geheimnis dieser Linien zu liegen, sie sind für sich autonom und nicht gegenstandsbezogen, gewinnen aber im Kontext Verweiskraft und tendenziell Zeichenhaftigkeit zurück, wobei sie allerdings auf unsere Projektion angewiesen sind.

Die späten Zeichnungen von Rembrandt, die sich biblischer oder mythologischer Geschichte widmen, sind erstaunlich bildmäßig angelegt, der Künstler verstärkt diesen Eindruck häufig noch, indem er um die Darstellung mit groben Strichen einen Rahmen zeichnet. Die aller Sperrigkeit zum Trotz hochdifferenzierten Einsatzmöglichkeiten der Rohrfeder seien abschließend an der Dresdener Zeichnung „Petrus am Totenbett der Tabea" (Abb. 16), die bereits in die sechziger Jahre und damit in die letzte Phase von Rembrandts Kunst gehört, demonstriert. ${ }^{38}$ Der Dresdener Katalog beschreibt die Technik als: „Feder in Braun, Pinsel, gewischt, Deckweiß“. ${ }^{39}$ Darin scheint mir der Hinweis auf den Pinsel nicht richtig zu sein. Es dürfte sich um eine reine Rohrfederzeichnung handeln. Nimmt man mit der Rohrfeder viel Farbe auf und zeichnet dann mit starkem Druck, so gibt sie viel und ungleichmäßig Farbe ab, die oben an den Fransen des Baldachins über der toten Tabea gewischt ist und einen Flächenwert bildet, gelegentlich macht Rembrandt dies mit den Fingern. ${ }^{40}$ Dass die einzelnen dicken und erst am Ende eingetragenen Fransen der Rohrfeder entstammen, machen die schmalen zwischen manchen Fransen vorhandenen Verbindungslinien deutlich. Bei den Fransen ist die Feder gerade herunter gezogen worden mit dem Einschnitt in den Kiel in der Mitte, die Verbindungslinie zur nächsten Franse geschieht in verkanteter Federhaltung, so dass nur die eine Hälfte des gespaltenen Rohrs und auch nur mit dem Rand zeichnet. Die Rohrfeder ist nämlich durchaus in der Lage, bei Bedarf feine Linien zu zeichnen. Sie darf dabei nicht zuviel Farbe transportieren und muss besonders schräg gehalten werden, damit sie nur mit der selbst nicht Farbe tragenden Kante zeichnet. Rembrandt nutzt diese Möglichkeiten regelmäßig für feine Parallelschraffuren, um Schatten anzudeuten, hier gut erkennbar bei dem von Petrus aus dem Raum verwiesenen Trauerweib ganz rechts.

38 Kat.Ausst. Rembrandt. Die Dresdener Zeichnungen (2004) Kat. Nr. 116.

39 Ebenda 203.

40 S. Kat. Rembrandt auf Papier (2001) 35 (Peter Schatborn); Kat. Tekeningen van Rembrandt (1985) Kat. Nr. 54; vom Verreiben mit dem Finger spricht bereits: van Hoogstraten (1678) 31. 
Wie ist der Werdegang einer solchen Zeichnung zu denken? Wohl nicht so viel anders als bei der früheren Zeichnung „Saskia im Bett mit einer Amme". Am feinsten ist die tote Tabea im Bett gezeichnet, offenbar nur hier ist auch Deckweiß zum Einsatz gekommen, wohl weniger um ihre Haube zu korrigieren, als sie in der Farbstärke zurückzunehmen, dieser Partie ihre Feinheit zu lassen. Da Deckweiß oxidiert, sind abgedeckte Linien heute leicht zu erkennen. Dann kam die Gruppe mit Petrus und zwei Frauen, von denen die eine sich bereits zum Gehen gewendet hat, während die andere links von Petrus noch dasteht und sich zurückwendet. Die dritte Figur scheint nachträglich über das Gestell des Baldachins mit besonders starken Strichen gezeichnet worden zu sein, sie wendet uns wohl den Rücken zu und steht noch unentschlossen da. Petrus jedoch verweist alle energisch des Raumes, die wundersame Wiedererweckung soll ohne Zuschauer vonstatten gehen. Zum Schluss, auch das behält Rembrandt aus der Frühzeit bei, sind die verschattenden Schraffuren in unterschiedlicher Dichte und Feinheit angebracht worden, über allem jedoch liegen die groben Fransen als letzte Zutat. Ihr dunkler Ton steigert die Helligkeit über der Toten und gibt ihr, wie die zarte Zeichnung, Ruhe. Die kubischen Kompartimente, die geradezu geometrische Vereinfachung des Figurenbaus stiften erneut Monumentalität. Die blockhafte Gruppe der vier stehenden Figuren, bei denen durchaus unklar ist, welcher Teil zwingend zu welcher Person gehört, ist, denkt man sich allein die Köpfe weg, schier unlesbar und doch ist das durch das Gewirr hindurch sich abzeichnende Geschehen seiner Tendenz nach eindeutig. Keine Linie, nicht eine, ist schönheitlich und doch ist die Würde des Geschehens eingefangen. Ein wahres Wunderwerk ist der Kopf des Petrus, er macht ihn als Petrus unmittelbar erkennbar und das obwohl die groben Striche für sich nichts bezeichnen. Die hohe Stirn, der kurze Backenbart mit den geteilten Kompartimenten links und rechts, die schwindenden Kopfhaare, das eher gröbere Gesicht mit der dickeren kurzen Nase unter dichten Brauen - Rembrandt beherrschte den überlieferten Petrustypus im Schlaf, und dennoch ist es erstaunlich, wie er ihn der krakeligen Rohrfeder abgewonnen hat.

Die kubische Sprache ist auch eine Kürzelsprache und zeichnerische Kürzel hat Rembrandt Zeit seines Lebens erprobt und an seine zahlreichen Schüler weitergegeben. ${ }^{41}$ So wird man sagen müssen, dass ihm die Kürzelsprache zur punktuellen genaueren Charakterisierung verhilft, hier macht sie das Thema zusammen mit dem Weisegestus unmittelbar identifizierbar

41 Zum erlernbaren Rembrandtstil, der offenbar mit körpermotorischen Bewegungen eingeübt wurde: Alpers (1989) Kap. 3 Ein Meister im Atelier, 128-197. 
und lässt doch dem Betrachter genug Raum, um die Geschichte fortzuspinnen. Räumlichkeit ist nicht nachmessbar angegeben, doch evoziert, die Federstärke ruft die Evokation hervor, die gleichzeitige Unbestimmtheit des einzelnen Striches, die Signifikation dieses Unbestimmten durch den Zusammenhang mit anderen Strichen zeigt uns, wie Zeichenhaftigkeit im künstlerischen Prozess sich einstellt. Rembrandt demonstriert uns die Alternative zum idealistischen Formkonzept: Kunst als subjektiv bedingter Entstehungsprozess, der anschaulich bleibt, gegen Kunst als ideale Objektivation.

\section{Literaturverzeichnis}

Alpers, Svetlana, The Art of Describing. Dutch Art in the Seventeenth Century, Chicago 1983.

Dies, Rembrandt als Unternehmer. Sein Atelier und der Markt, Köln 1989 (zuerst engl. als „Rembrandt's Enterprise“, Chicago 1988).

Courtright, Nicola, Origins and Meanings of Rembrandt's late Drawing Style, in: The Art Bulletin 78, 1996, 485-510.

van Eikema Hommes, Margriet und van de Wetering, Ernst, Licht und Farbe bei Caravaggio und Rembrandt - mit den Augen ihrer Zeitgenossen gesehen, in: Kat.Ausst. Rembrandt-Caravaggio, hg. von Duncan Bell, Rijksmuseum Amsterdam und van Gogh Museum Amsterdam, Zwolle 2006, 164-79.

Frey, Dagobert, Das Fragmentarische als das Wandelbare bei Rembrandt, in: Josef Adolf Schmoll gen. Eisenwerth (Hg.), Das Unvollendete als künstlerische Form, Bern 1959, 91-116.

Heublein, Brigitte, Der „,verkannte“ Joseph: zur mittelalterlichen Ikonographie des Heiligen im deutschen und niederländischen Kulturraum, Weimar 1998.

van Hoogstraten, Samuel, Inleyding tot de Hooge Schoole der Schilder-Konst..., Rotterdam 1678.

de Jongh, Eddy, Bespr. von Bob Haak, Hollandse schilders in de Gouden Eeuw, in: Simiolus 15, 1985, 65-68.

Kat.Ausst. Der späte Tizian und die Sinnlichkeit der Malerei, hg. von Sylvia Ferino-Pagden, Wien, Kunsthistorisches Museum und Venedig, Gallerie dell'Accademia, Wien 2007.

Kat.Ausst. Rembrandt-Caravaggio, hg. von Duncan Bell, Rijksmuseum Amsterdam und van Gogh Museum Amsterdam, Zwolle 2006.

Kat.Ausst. Rembrandt. Der Meister und seine Werkstatt. Zeichnungen und Radierungen, hg. von Holm Bevers, Peter Schatborn und Barbara Welzel, Kupferstichkabinett, Staatliche Museen Preussischer Kulturbesitz; Rijksmuseum Amsterdam; The National Gallery London, München 1991. 
Kat.Ausst. Rembrandt. Die Dresdener Zeichnungen, hg. von Christian Dittrich und Thomas Ketelsen, Staatliche Kunstsammlungen Dresden, KupferstichKabinett, Köln 2004.

Kat.Ausst. Rembrandt. Die Opferung Isaaks, hg. von Marcus Dekiert, Alte Pinakothek, Bayerische Staatsgemäldesammlungen, München 2004.

Kat.Ausst. Rembrandt. Genie auf der Suche, Gemäldegalerie, Staatliche Museen zu Berlin, Köln 2006.

Kat.Ausst. Rembrandt Rembrandt, Städelsches Kunstinstitut und Städtische Galerie Frankfurt am Main, Kyoto National Museum, Wolfratshausen 2003.

Kat.Ausst. Rembrandt the Printmaker, hg. von Erik Hinterding, Ger Luijten and Martin Royalton-Kisch, The British Museum, London 2000.

Kat. Drawings by Rembrandt and his Circle in the British Museum, hg. von Martin Royalton-Kisch, London 1992.

Kat. Rembrandt auf Papier. Werk und Wirkung, hg. von Thea Vigneau-Wilberg, Staatliche Graphische Sammlung München, München, Alte Pinakothek, Amsterdam, Museum het Rembrandthuis, München 2001

Kat. Tekeningen van Rembrandt, zijn onbekende leerlingen en navolgers Drawings by Rembrandt, his anonymous pupils and followers, hg. von $\mathrm{Pe}-$ ter Schatborn, Rijksprentenkabinet, Rijksmuseum Amsterdam, s'Gravenhage 1985.

Krüger, Klaus, Innerer Blick und ästhetisches Geheimnis: Caravaggios „Magdalena“, in: Joseph Imorde, Fritz Neumeyer und Tristan Weddigen (Hgg.), Barocke Inszenierung, Emsdetten/Zürich 1999, 33-49.

Kroschewski, Nevenka, Über das allmähliche Verfertigen der Bilder. Neue Aspekte zu Caravaggio, München 2002.

Rosand, David, Drawing Acts. Studies in Graphic Expression and Representation, Cambridge 2002.

Royalton-Kisch, Martin, Rembrandt's Sketches for his Paintings, in: Master Drawings $27,1989,128-145$.

Ders., The role of drawings in Rembrandt's printmaking, in: Kat.Ausst. Rembrandt the Printmaker, hg. von Erik Hinterding, Ger Luijten and Martin Royalton-Kisch, The British Museum, London 2000, 64-81.

Smith, David R., „I Janus": Privacy and the gentlemanly Ideal in Rembrandt's Portraits of Jan Six, in: Art History 2, 1988, 42-63.

Taylor, Paul, The Concept of Houding in Dutch Art Theory, in: Journal of the Warburg and Courtauld Institutes 55, 1992, 210-232.

Tümpel, Christian, Pieter Lastman en Rembrandt - Pieter Lastmann and Rembrandt, in: Kat.Ausst. Pieter Lastman. Leermeester van Rembrandt - The man who taught Rembrandt, hg. von Astrid Tümpel und Peter Schatborn, Het Rembrandthuis Amsterdam, Zwolle 1991, 54-84.

Waiboer, Adriaan E., Lastmans Opferdarstellungen und ihre weitreichende Wirkung, in: Kat.Ausst. Pieter Lastman. In Rembrandts Schatten, hg. von Martina Sitt, Hamburger Kunsthalle, München 2006.

van de Wetering, Ernst, Rembrandt. The Painter at Work, Amsterdam 2000. 


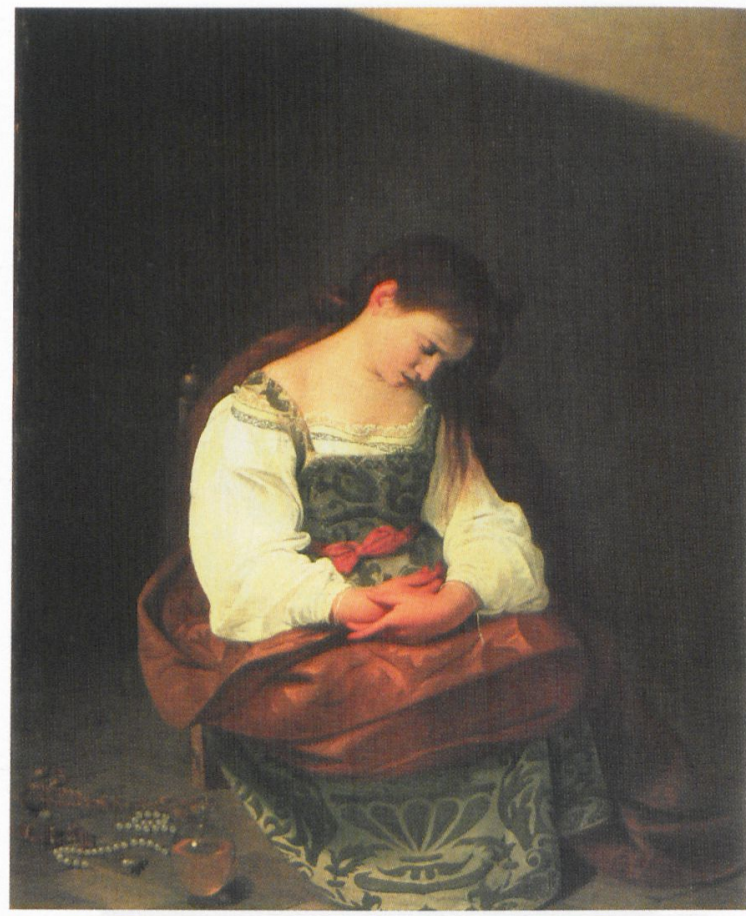

Abbildung 1. Caravaggio, Büßende Maria Magdalena, 1598

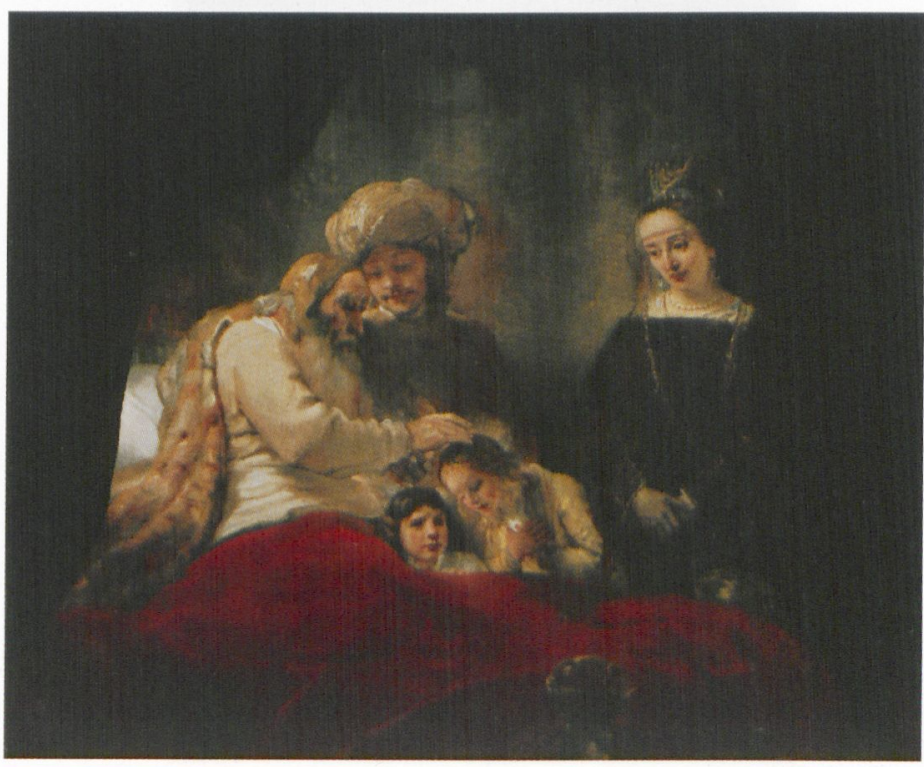

Abbildung 2. Rembrandt, Jakobssegen, 1656 


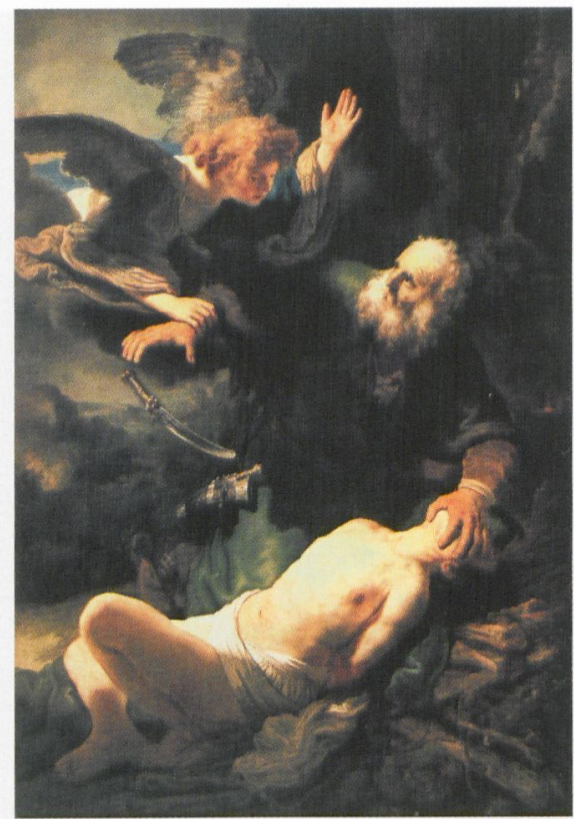

Abbildung 3. Rembrandt, Abrahamsopfer, 1635

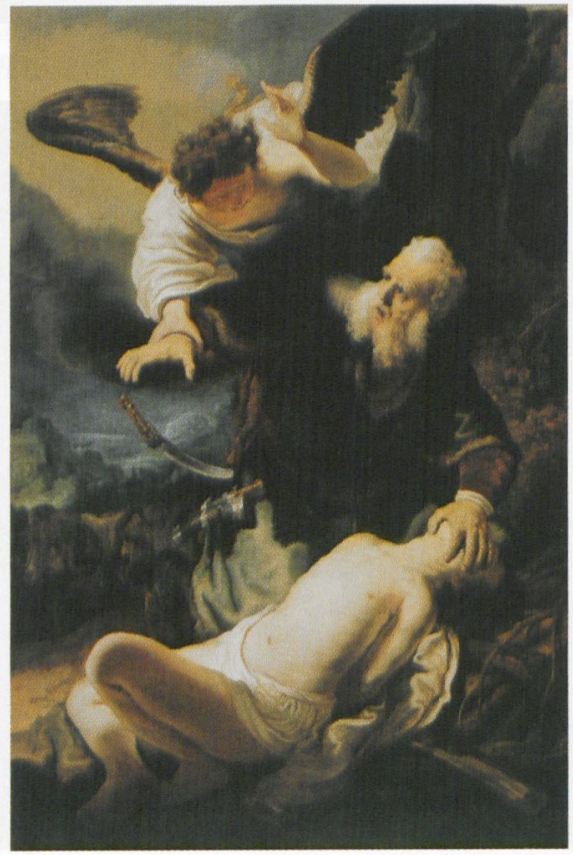

Abbildung 4. Rembrandt und Werkstatt, Abrahamsopfer, 1636 


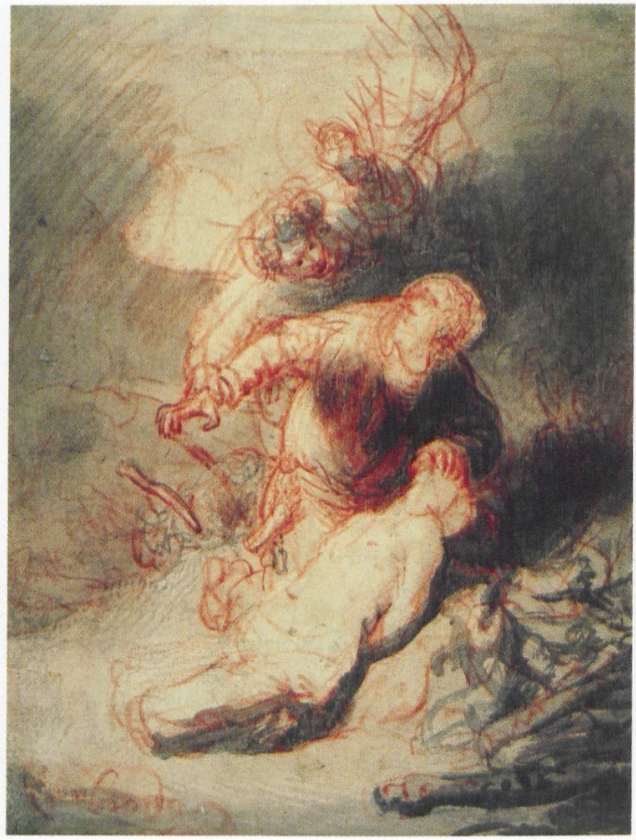

Abbildung 5. Rembrandt, Abrahamsopfer

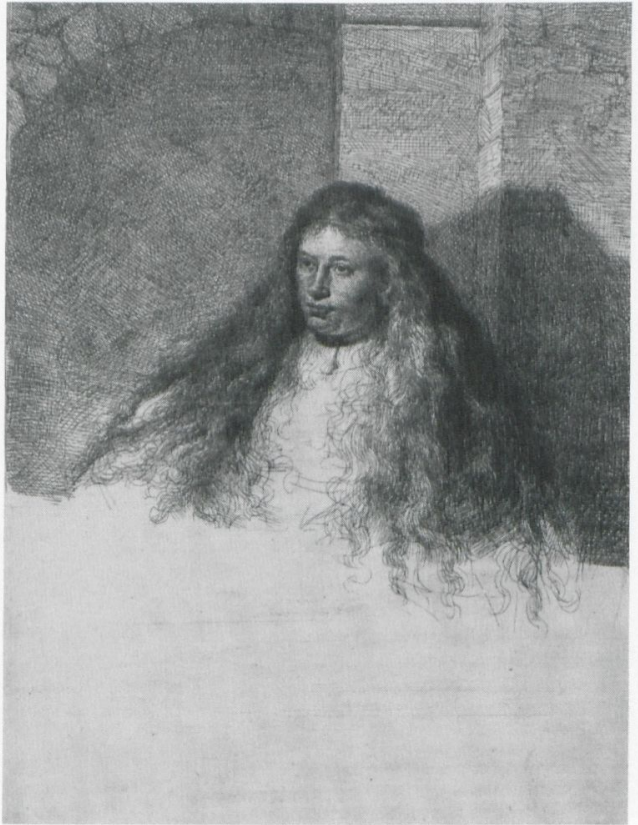

Abbildung 6. Rembrandt, Große Judenbraut, 1635 


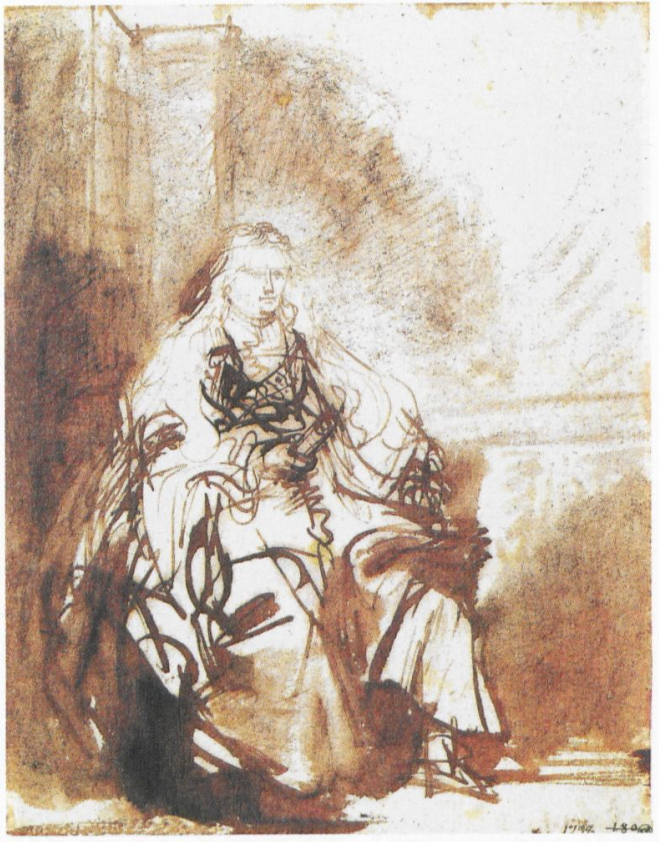

Abbildung 7. Rembrandt, Zeichnung zur Großen Judenbraut

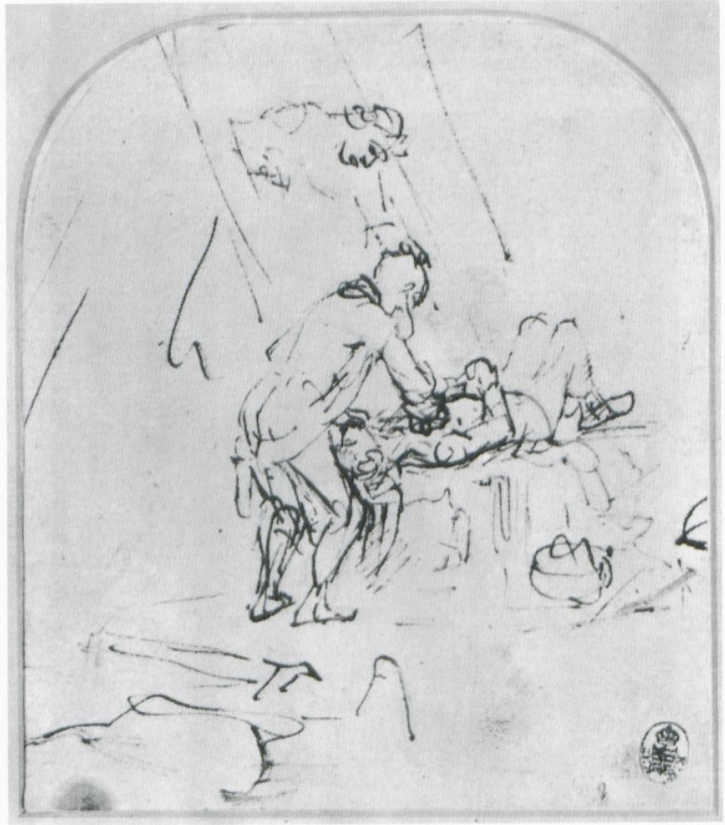

Abbildung 8. Rembrandt, Abrahamsopfer, 1652-1654 


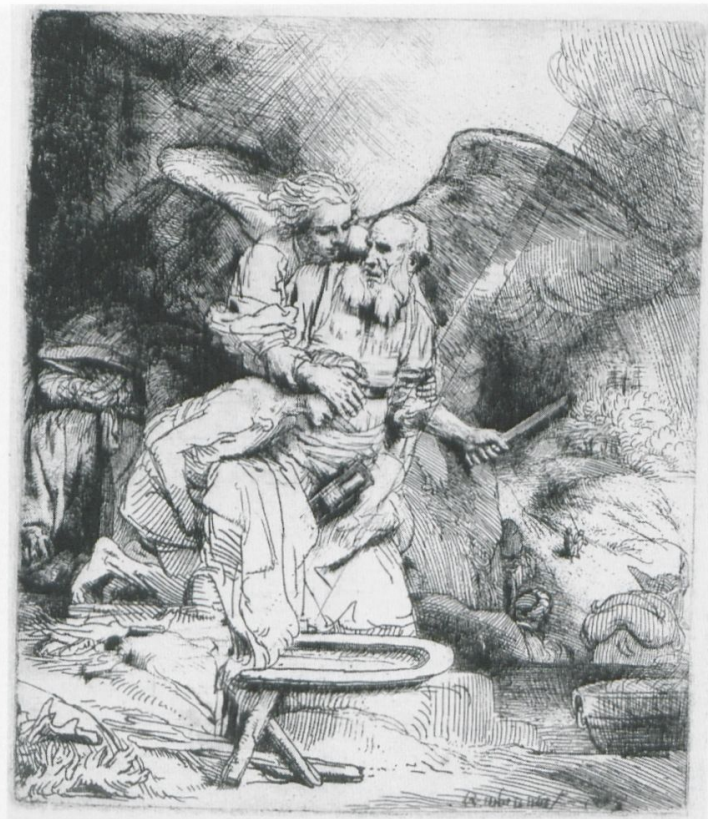

Abbildung 9. Rembrandt, Abrahamsopfer, 1655

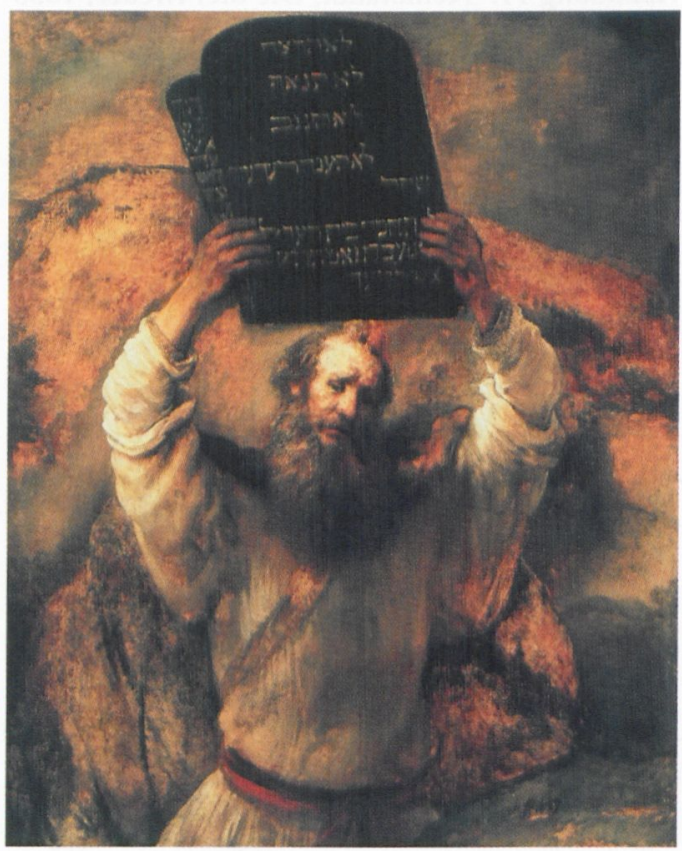

Abbildung $\cdot 10$. Rembrandt, Moses, 1659 


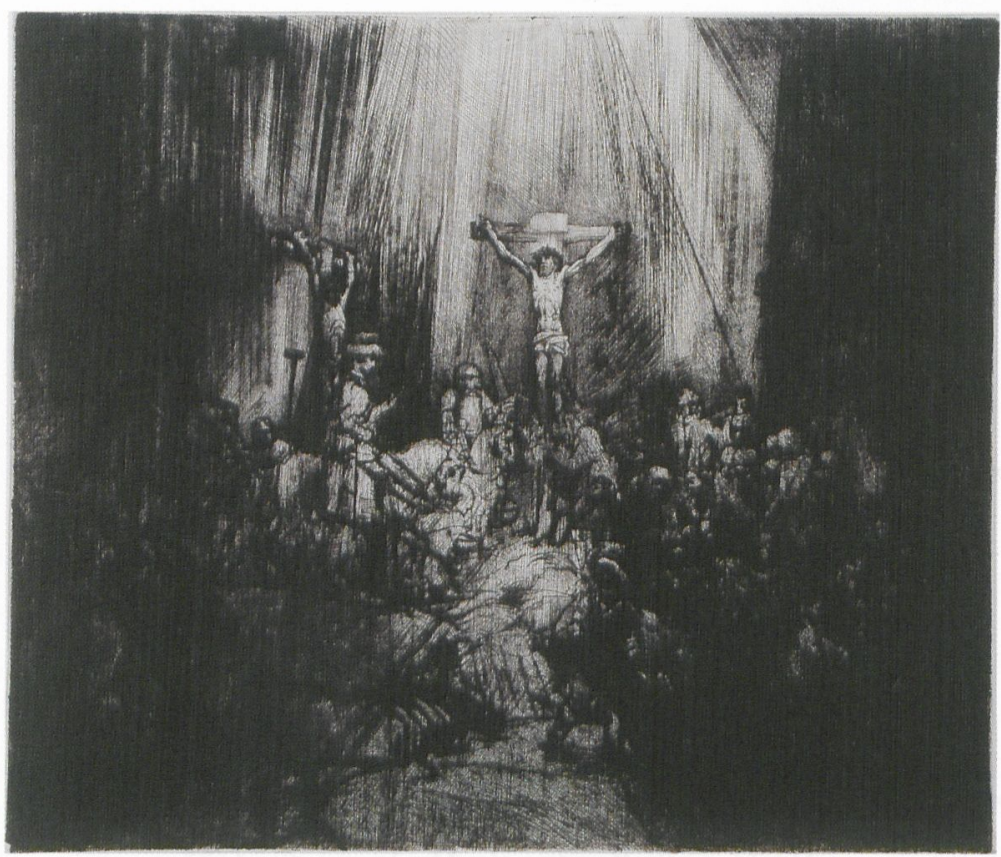

Abbildung 11. Rembrandt, Die drei Kreuze, 1653

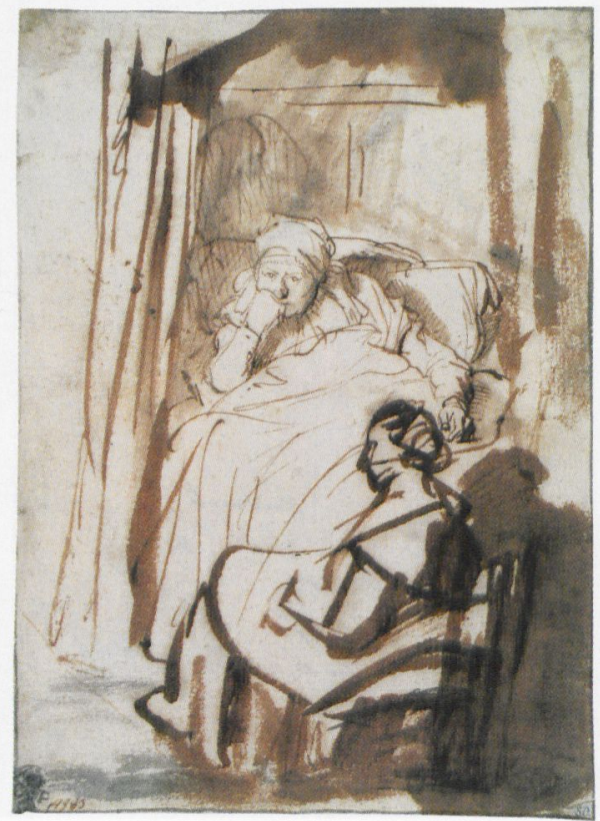

Abbildung 12. Rembrandt, Saskia im Bett mit einer Amme, um 1638 


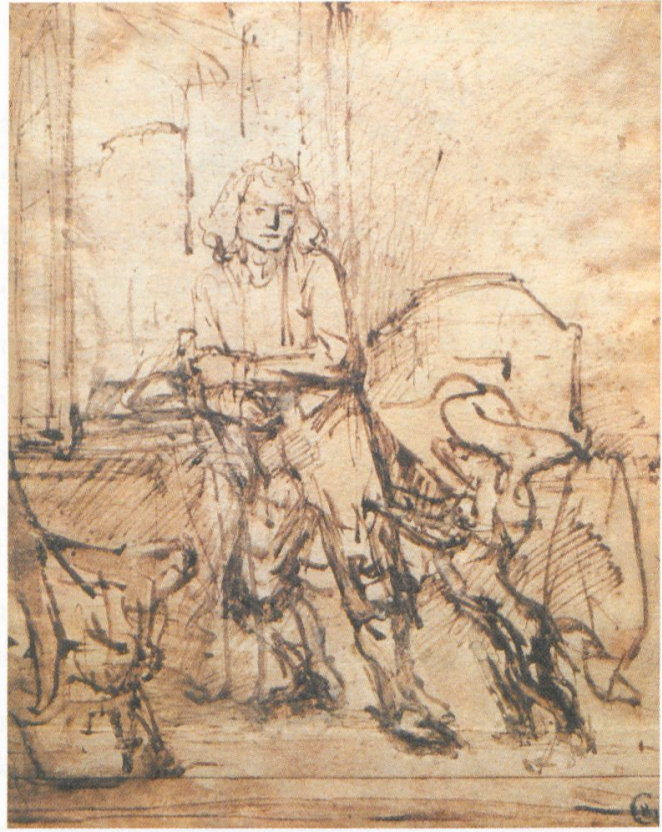

Abbildung 13. Rembrandt, Jan Six

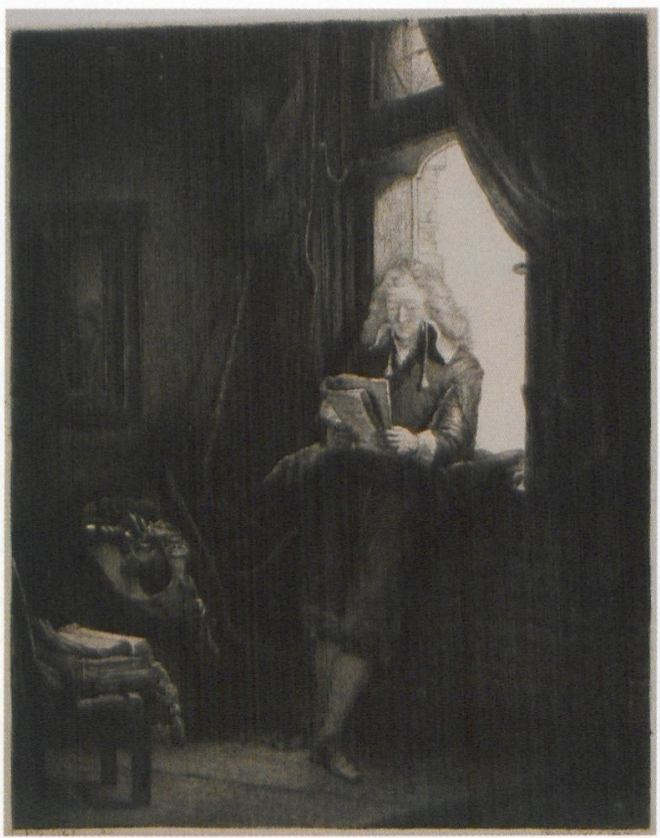

Abbildung 14. Rembrandt, Jan Six, Radierung, 1647 


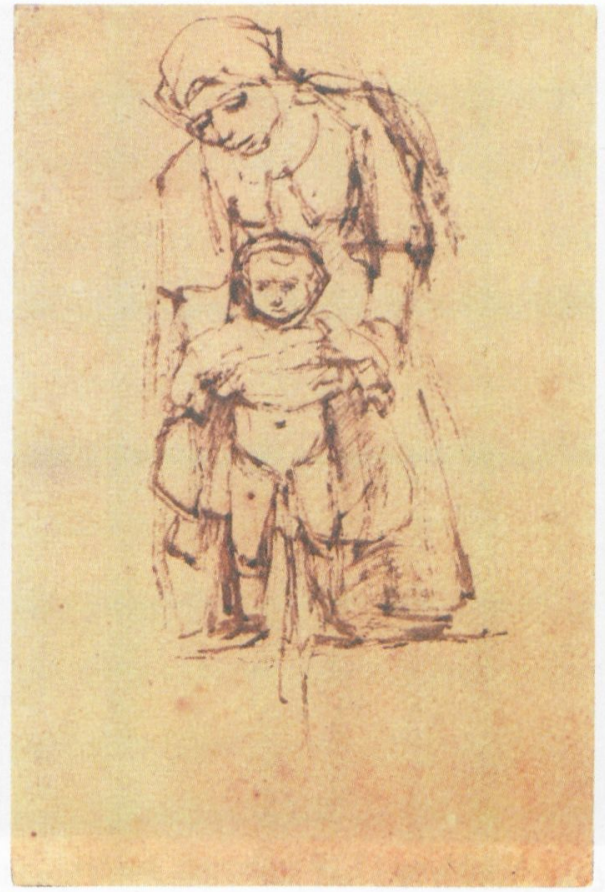

Abbildung 15. Rembrandt, Frau mit pissendem Kind, um 1657-1660

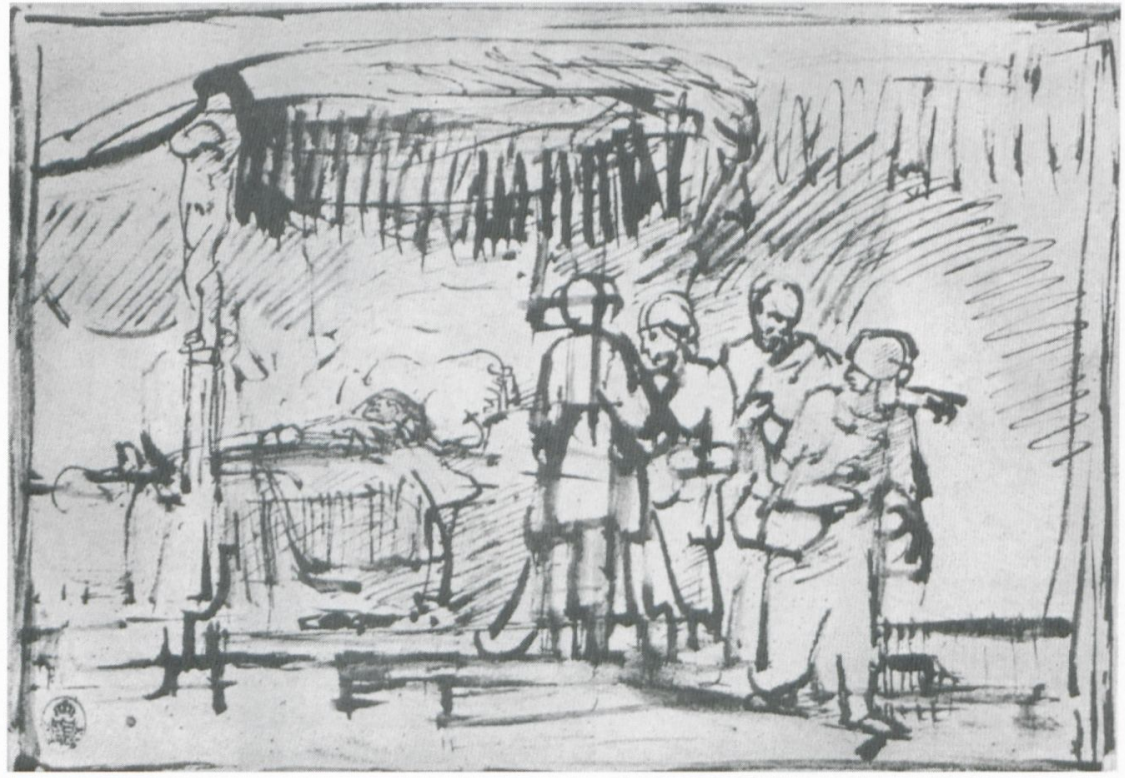

Abbildung 16. Rembrandt, Petrus am Totenbett der Tabea, um 1662-1665 


\section{Abbildungsverzeichnis}

Abb. 1: Caravaggio, Büßende Maria Magdalena, 1598, Öl auf Leinwand, 122,5 × 98,5 cm, Rom, Galleria Doria Pamphilj

Abb. 2: Rembrandt, Jakobssegen, 1656, Öl auf Leinwand, $175,0 \times 210,5 \mathrm{~cm}$, Kassel, Gemäldegalerie

Abb. 3: Rembrandt, Abrahamsopfer, 1635, Öl auf Leinwand, $193,0 \times 133 \mathrm{~cm}$, St. Petersburg, Eremitage

Abb. 4: Rembrandt und Werkstatt, Abrahamsopfer, 1636, Öl auf Leinwand, 195,0 × 132,3 cm, München, Bayer. Staatsgemäldesammlungen, Alte Pinakothek

Abb. 5: Rembrandt, Abrahamsopfer, rote und schwarze Kreide mit Pinsellavierung, 19,5 × 14,7 cm, London, British Museum

Abb. 6: Rembrandt, Große Judenbraut, 1635, Radierung, 21,9 × 16,8 cm, 2. Zustand, Amsterdam, Rijksmuseum

Abb. 7: Rembrandt, Zeichnung zur Großen Judenbraut, Feder in Braun mit Lavierung, 24,0 × 19,0 cm, Stockholm, Nationalmuseum

Abb. 8: Rembrandt, Abrahamsopfer, 1652-1654, Feder in Braun, $18,0 \times 15,5 \mathrm{~cm}$, Dresden, Kupferstich-Kabinett

Abb. 9: Rembrandt, Abrahamsopfer, 1655, Radierung, 15,6 6 13,1 cm, Amsterdam Rijksmuseum

Abb. 10: Rembrandt, Moses, 1659, Öl auf Leinwand, 168,5 × 136,5 cm, Berlin, Staatl. Museen, Gemäldegalerie

Abb. 11: Rembrandt, Die drei Kreuze, 1653, Kaltnadel, 38,5 × 45,0 cm, 4. Zustand, London, British Museum

Abb. 12: Rembrandt, Saskia im Bett mit einer Amme, um 1638, Feder und Pinsel in Braun laviert, 22,7 × 16,4 cm, München, Staatl. Graphische Sammlung

Abb. 13: Rembrandt, Jan Six, Entwurfszeichnung für die Radierung, Feder in Braun, laviert mit weißen Korrekturen, 22,0 × 17,7 cm, Amsterdam, Six Collection

Abb. 14: Rembrandt, Jan Six, 1647, Radierung, 24,4 × 19,1 cm, 4. Zustand, London, British Museum 
Abb. 15: Rembrandt, Frau mit pissendem Kind, um 1657-1660, Feder in Braun, 13,3 × 9,0 cm, Dresden, Kupferstich-Kabinett

Abb. 16: Rembrandt, Petrus am Totenbett der Tabea, um 1662-1665, Feder in Braun, mit Weiß korrigiert, 19,0 × 27,3 cm, Dresden, Kupferstich-Kabinett

Sämtliche Abbildungen aus dem Bildarchiv des Kunsthistorischen Instituts, Freie Universität Berlin 